\title{
EVAPOTRANSPIRAÇÃO DE REFERÊNCIA PARA DIMENSIONAMENTO DE SISTEMAS DE IRRIGAÇÃO EM PETROLINA, PE
}

\author{
A. O. da Silva ${ }^{1}$; J. de S. Correia ${ }^{1}$; L. H. Bassoi ${ }^{2}$; A. H. de C. Teixeira ${ }^{3}$ \\ ${ }^{1}$ Faculdade de Ciências Agronômicas, UNESP - Univ Estadual Paulista, Botucatu, SP, Brasil \\ ${ }^{2}$ Empresa Brasileira de Pesquisa Agropecuária, EMBRAPA - Semiárido, Petrolina, PE, \\ Brasil \\ ${ }^{3}$ Empresa Brasileira de Pesquisa Agropecuária, EMBRAPA -Monitoramento por satélites, \\ Campinas, SP, Brasil.
}

\section{RESUMO}

A evapotranspiração é uma variável fundamental para estimar a exigência hídrica das culturas, e também fornece subsídio para o dimensionamento de sistemas de bombeamento, adução e distribuição de água na irrigação. A caracterização da distribuição de frequência da evapotranspiração de referência (ETo), estimada pelo método do tanque classe "A" em Petrolina, PE, foi realizada com base em série histórica de 45 anos (1963 a 2010) de dados, os quais foram agrupados em períodos de 5, 10, 15 e 30 dias. As distribuições Gama e Normal foram aplicadas aos dados de ETo com probabilidade de 95, 90, 75, 50, 25, 10 e 5\%. Sendo aptas para representar os dados de evapotranspiração de referência para os períodos acumulados de 10, 15 e 30 dias. Os valores de ETo $\left(11,06\right.$ e $11,07 \mathrm{~mm}$ dia $^{-1}$, distribuição gama e normal, respectivamente) para o mês de outubro no período acumulado de 15 dias com probabilidade de $75 \%$, foram os mais indicados para o dimensionamento da lâmina diária máxima de projetos de sistemas de irrigação.

Palavras-chave: Probabilidade, frequência de distribuição, semiárido.

\section{REFERENCE EVAPOTRANSPIRATION FOR DESIGN OF IRRIGATION SYSTEMS IN PETROLINA, BRAZIL}

\begin{abstract}
Evapotranspiration is a key variable to estimate crop water requirement, to support the design of pumping systems, supply and distribution of water for irrigation. The distribution frequence of reference evapotranspiration (ETo) estimated by class A pan in Petrolina, State of Pernambuco, Brazil, was characterized based on a series of 45 years (1963 - 2010), grouped in periods of 5,10,15 and 30 days. Gamma and Normal distributions were applied to the ETo data with a probability of 95, 90, 75, 50, 25, 10 and 5\%. The Normal and gamma distributions were able to represent the data of reference evapotranspiration for the cumulative periods of 10, 15 and 30 days. The ETo values (11.06 and $11.07 \mathrm{~mm} \mathrm{day}^{-1}$, normal and gamma distribution, respectively) for the month of October in the period from 15 days with $75 \%$ probability were the most suitable for the scaling of the maximum daily depth projects of irrigation systems.
\end{abstract}

Keywords: Probability, distribution frequency, semi-arid.

\footnotetext{
"alexsandro_oliveira01@hotmail.com
} 


\section{INTRODUÇÃO}

A agricultura apresenta grande dependência das condições climáticas atuantes nas regiões de cultivo, sendo influenciada de maneira significativa pelos diversos fatores meteorológicos existentes, dentre estes fatores destacam-se a precipitação, radiação solar, vento, evapotranspiração e etc. Por isso, é fundamental estudar a influência das suas variações sobre as diferentes estratégias de uso do sistema agrícola, de modo a apresentar subsídios para o processo de tomada de decisão e otimizar o planejamento das atividades agrícolas (FRIZZONE \& ANDRADE JÚNIOR, 2005).

$\mathrm{Na}$ agricultura irrigada, o conhecimento da evapotranspiração é de grande importância na estimativa das necessidades hídricas das culturas, bem como para o manejo racional dos recursos hídricos (ABABAEI, 2014; CARVALHO $\&$ OLIVEIRA, 2012). Segundo SAAD et al. (2002) a estimativa da necessidade hídrica das culturas é de fundamental importância para dimensionar o sistema de adução, de distribuição e de aplicação de água, característicos de cada método de irrigação. Porém, existem dificuldades para uma estimativa adequada da evapotranspiração da cultura, pois a diversidade de métodos de estimativa da evapotranspiração de referência (ETo) e a dificuldade para seu cálculo, devido a grande quantidade de parâmetros meteorológicos exigidos, além de breves históricos destes na maioria das propriedades, dificultam uma estimativa mais precisa. Por isso, a utilização de métodos alternativos para estimativa da ETo como o tanque classe "A" devem ser utilizados para o planejamento da agricultura irrigada, devido principalmente a sua fácil aplicação, além de possuir em alguns casos, dados históricos maiores, atribuídos principalmente ao período longo de uso das estações meteorológicas convencionais no Brasil (PEIXOTO et al. 2014; TRAJKOVIC \& KOLAKOVIC, 2010).
A utilização de simulações com o uso de dados históricos para o planejamento da irrigação é uma técnica adotada com a finalidade de fazer previsões de fenômenos meteorológicos, consistindo em simular uma ocorrência futura de possíveis valores (FIETZ et al. 1997). Segundo FRIZZONE \& ANDRADE JÚNIOR (2005) a técnica de simulação é de grande importância, devido às séries de dados climáticos disponíveis para efetuar estudos envolvendo as interrelações clima versus sistema agrícola serem muito pequenas, levando a obtenção de resultados tendenciosos. DOORENBOS \& PRUITT (1997) advertem que o uso de dados climáticos médios em equações combinadas podem conduzir a erros significativos, comprometendo todo planejamento realizado.

ASSIS et al. (2014), BACK (2007), SAAD et al. (2002) sugerem uma análise da probabilidade de ocorrência dos valores históricos de ETo para fins de dimensionamento de irrigação, porém estes autores afirmam que a escolha do nível de probabilidade deve basear-se em uma análise econômica, considerando os prejuízos associados à redução da quantidade e da qualidade da produção, decorrentes da deficiência hídrica, e o aumento de custos do sistema para satisfazer níveis mais elevados de probabilidade. DOOREMBOS \& PRUITT (1997) consideram que na maioria das regiões irrigadas os níveis de probabilidade estejam entre $75 \%$ e $80 \%$ sendo inviável economicamente a utilização de probabilidades superiores a $90 \%$, mesmo em condições semiáridas como o semiárido do Nordeste Brasileiro, que apesar do baixo regime de precipitação nesta região, apresenta épocas chuvosas nos primeiros meses do ano. O objetivo deste trabalho foi o de analisar a frequência e a distribuição dos dados de evapotranspiração de referência pelo método do tanque classe "A", na região de Petrolina, PE e seu ajustamento aos modelos probabilísticos Normal e Gama. 


\section{MATERIAIS E MÉTODOS}

Os dados utilizados foram obtidos de uma série de 53 anos (1960 a 2013) dos registros da estação meteorológica da área experimental da Embrapa Semiárido, localizada no perímetro irrigado de Bebedouro em Petrolina-PE (latitude $09^{\circ}$ $09^{\prime} \mathrm{S}$, longitude $40^{\circ} 22^{\prime} \mathrm{W}$ ) foram obtidos os dados diários de evaporação, velocidade do vento e umidade do ar. O clima da região é do tipo BSwh', segundo a classificação de Köppen, correspondente a uma região árida.

A evapotranspiração de referência (ETo) foi calculada pelo método do tanque classe "A" conforme equação 1 e descritos por DOORENBOS \& PRUITT (1997), nos 12 meses de cada ano estudado, após, foram obtidos os valores para os intervalos de cinco (pentadas), dez (decêndios), quinze (quinzenais) e trinta (mensais) dias para os valores de ETo.

$$
E T o=E C A \times K p
$$

Onde, ETo é a evapotranspiração de referência $\left(\mathrm{mm} \mathrm{dia}{ }^{-1}\right)$; ECA é a evaporação do tanque $\left(\mathrm{mm} \mathrm{dia}^{-1}\right)$; Kp é o coeficiente do tanque classe " $A$ " (adimensional).

Os valores do coeficiente do tanque para as condições locais de Petrolina, foram obtidos com base na tabela proposta por DOORENBOS \& PRUITT (1997) utilizando-se a equação de regressão ajustada por SNYDER (1992):

$$
\begin{aligned}
& K p=0,482+0,024 \ln F-0,000376 \times \\
& U+0,0045 \times U R
\end{aligned} \times
$$

Onde, F é a distância da área de bordadura em relação ao centro do tanque $(\mathrm{m})$; $\mathrm{U}$ é a velocidade do vento $\left(\mathrm{km} \mathrm{h}^{-1}\right)$; UR é a umidade relativa do ar (\%).

Para verificar a frequência dos valores de ETo, foram calculados os valores esperados de evapotranspiração de referência com probabilidades de $5 ; 10 ; 25$; 50; 75; 90 e 95\%, utilizando a distribuição normal e a distribuição gama, a função densidade de probabilidade para a distribuição gama pode ser verificada pela equação 3 (SILVA et al. 2010):

$$
\begin{aligned}
& f(x, \alpha, \beta)= \\
& \left\{\begin{array}{l}
\frac{1}{\beta^{\alpha} \Gamma(\alpha)} X^{\alpha-1} e^{-\frac{x}{\beta}} \\
0
\end{array}\right. \\
& x \geq 0
\end{aligned}
$$

As estimativas dos parâmetros $\alpha$ e $\beta$ foram efetuadas pelo método da máxima verossimilhança, por meio das equações 4 a 8 (SILVA et al., 2013):

$$
\begin{aligned}
& \alpha=\frac{1}{4 A}\left(1+\sqrt{1+\frac{4 A}{3}}\right) \\
& \beta=\frac{\bar{X}}{\alpha} \\
& A=\ln \overline{\mathrm{X}}-\mathrm{X}_{\mathrm{g}} \\
& \bar{X}=\frac{1}{N} \sum_{i=1}^{N} \mathrm{x}_{\mathrm{i}} \\
& X_{g}=\frac{1}{N} \sum_{i=1}^{N} \ln \left(\mathrm{x}_{\mathrm{i}}\right)
\end{aligned}
$$

Onde, $\alpha$ é o parâmetro de forma; $\beta$ é o parâmetro de escala da distribuição Gama, $\mathrm{X}$ é a média aritmética da amostra; $\mathrm{X}_{\mathrm{g}}$ é a média geométrica da amostra; $\mathrm{N}$ é $\mathrm{O}$ número de anos observados.

A função densidade de probabilidade da distribuição normal pode ser representada pela equação 9 (ARAÚJO et al. 2010):

$$
\underset{+\infty}{f(x)=\frac{1}{\sigma \sqrt{2 \pi}} e\left(-\frac{(x-\mu)^{2}}{2 \sigma^{2}}\right) ; \text { para } \infty<}
$$

Onde, $\mu$ é a média; $\sigma$ é o desvio padrão da variável aleatória.

Para verificar o ajuste das distribuições de probabilidade aos dados de evapotranspiração de referência, foi realizado o teste de Kolmogorov-Smirnov, a 5\% de significância, conforme equações 10, 11 e 12 (CHICHEPORTICHE \& 
BOUCHAUD, 2012), segundo Campos (1983) este teste verifica a adaptação de uma específica e bem conhecida distribuição a dados provenientes de uma distribuição desconhecida.

$D_{n}^{+}=\max \left(F_{n}(x)-F(x)\right)$

$D_{n}^{-}=\max \left(F(x)-F_{n}(x)\right)$

$$
F_{n}(x)=\frac{1}{n} \sum_{i=1}^{n}\left\{\begin{array}{l}
1, \text { se } y_{i}<x, \\
0, \text { Caso contrário }
\end{array}\right.
$$

Onde, $\mathrm{F}_{\mathrm{n}}(\mathrm{x})$ é a função distribuição acumulada para $n$ observações; $\mathrm{F}(\mathrm{x})$ é a função de distribuição em hipótese.

As analises probabilísticas, assim como os testes de aderência foram realizados utilizando as planilhas do Microsoft Excel ${ }^{\circledR}$ (CURTIS, 2012).

para redução dos erros, para que não comprometa a eficiência destes. Para o cálculo da distribuição gama os valores de $\alpha$ variaram entre 59,28 e 19,79 para o período chuvoso na região e entre 44,50 a 12,47 para o período de maior demanda hídrica para as culturas, os valores de $\beta$ apresentaram valores entre 3,94 e 19,24, os valores de $\beta$ foram inferiores a 100 possibilitando o uso destes para a distribuição estudada, pois segundo THOM (1958) valores de $\beta$ superiores a 100 não devem ser utilizados para a distribuição gama incompleta.

Tabela 1. Parâmetros estatísticos mensais da evapotranspiração de referência para o perímetro irrigado de Bebedouro-PE, no período entre 1960 e 2013

\begin{tabular}{|c|c|c|c|c|c|c|c|c|}
\hline \multirow{2}{*}{ Mês } & $\mu$ & $\mathrm{X}$ & $\sigma$ & Máxima & Mínima & $\alpha$ & $\mathrm{B}$ & \multirow{2}{*}{$\frac{\mathrm{CV}}{\%}$} \\
\hline & \multicolumn{5}{|c|}{ |--------------------------- mm ----------------------- } & & & \\
\hline Janeiro & 220,99 & 221,60 & 37,74 & 309,22 & 135,04 & 33,72 & 6,55 & 17,0 \\
\hline Fevereiro & 190,19 & 184,87 & 36,17 & 282,02 & 127,04 & 29,25 & 6,50 & 19,0 \\
\hline Março & 187,37 & 187,62 & 35,47 & 300,50 & 126,4 & 29,12 & 6,43 & 19,0 \\
\hline Abril & 172,83 & 169,40 & 38,19 & 255,30 & 77,80 & 19,79 & 8,73 & 22,0 \\
\hline Maio & 179,06 & 178,70 & 33,05 & 249,37 & 109,5 & 29,81 & 6,01 & 18,0 \\
\hline Junho & 175,24 & 173,98 & 26,40 & 234,61 & 122,66 & 44,50 & 3,94 & 15,0 \\
\hline Julho & 196,19 & 196,94 & 28,52 & 257,41 & 118,74 & 45,49 & 4,31 & 15,0 \\
\hline Agosto & 239,80 & 246,60 & 39,78 & 302,60 & 18,16 & 12,47 & 19,24 & 17,0 \\
\hline Setembro & 263,56 & 271,36 & 41,48 & 304,20 & 96,30 & 25,64 & 10,28 & 16,0 \\
\hline Outubro & 288,69 & 300,34 & 45,47 & 364,30 & 114,5 & 32,67 & 8,84 & 16,0 \\
\hline Novembro & 264,27 & 268,24 & 33,73 & 322,01 & 192,4 & 59,28 & 4,46 & 13,0 \\
\hline Dezembro & 233,11 & 240,38 & 43,95 & 311,50 & 153,08 & 27,68 & 8,42 & 19,0 \\
\hline
\end{tabular}

média $(\mu)$, mediana $(X)$, desvio-padrão $(\sigma)$, coeficiente de variação $(\mathrm{CV})$

Na Tabela 2, encontram-se os valores dos desvios máximos entre os valores observados e os valores estimados de evapotranspiração de referência para a distribuição normal e gama. Observa-se que as distribuições estudadas no período de 5 dias não apresentaram ajuste para a maioria dos meses em estudo, rejeitando-se assim a hipótese Ho ao nível de significância de 5\%. Para os demais 
períodos (10, 15 e 30 dias) as distribuições de frequências estudadas apresentaram um ajuste adequado para o teste de aderência proposto, aceitando-se assim a hipótese Ho para estas condições. Segundo SILVA et al. (1998), em estudos sobre a distribuição e frequência da evapotranspiração em Cruz das Almas-BA, devido a semelhança entre os ajustes das distribuições de frequência com relação ao teste de aderência, estes autores recomendam o uso da distribuição normal, devido a sua maior facilidade de aplicação. Porém, alguns autores como BACK (2007) e Saad et al. (2002) recomendam o uso da distribuição beta pois esta apresentou melhores ajustes para as regiões de Urusanga-SC e PiracicabaSP.

Tabela 2. Desvios máximos entre as frequências observadas e as frequências estimadas pela distribuição normal e distribuição gama para valores de evapotranspiração de referência em Petrolina-PE

\begin{tabular}{lllllllll}
\hline \multirow{2}{*}{ Mês } & \multicolumn{7}{c}{ Intervalos (dias) } \\
\cline { 2 - 9 } & \multicolumn{2}{c}{5} & \multicolumn{2}{c}{10} & \multicolumn{2}{c}{15} & \multicolumn{2}{c}{30} \\
\cline { 2 - 9 } Jan & $0,074^{*}$ & 0,122 & $0,041^{*}$ & $0,054^{*}$ & $0,080^{*}$ & $0,075^{*}$ & $0,098^{*}$ & $0,085^{*}$ \\
Fev & 0,101 & $0,086^{*}$ & 0,103 & $0,070^{*}$ & $0,066^{*}$ & $0,082^{*}$ & $0,142^{*}$ & $0,070^{*}$ \\
Mar & 0,098 & $0,075^{*}$ & $0,082^{*}$ & $0,092^{*}$ & $0,056^{*}$ & $0,098^{*}$ & 0,679 & 0,689 \\
Abr & $0,089^{*}$ & $0,064^{*}$ & $0,090^{*}$ & $0,060^{*}$ & $0,084^{*}$ & $0,093^{*}$ & $0,085^{*}$ & $0,070^{*}$ \\
Mai & 0,102 & $0,066^{*}$ & $0,035^{*}$ & $0,070^{*}$ & $0,068^{*}$ & $0,043^{*}$ & $0,101^{*}$ & $0,092^{*}$ \\
Jun & $0,079^{*}$ & $0,055^{*}$ & $0,048^{*}$ & $0,071^{*}$ & $0,062^{*}$ & $0,062^{*}$ & $0,081^{*}$ & $0,070^{*}$ \\
Jul & 0,127 & 0,093 & $0,042^{*}$ & $0,055^{*}$ & $0,074^{*}$ & $0,079^{*}$ & $0,055^{*}$ & $0,064^{*}$ \\
Ago & 0,151 & 0,133 & $0,065^{*}$ & $0,086^{*}$ & $0,092^{*}$ & $0,119^{*}$ & 0,247 & 0,350 \\
Set & 0,207 & 0,178 & $0,090^{*}$ & $0,105^{*}$ & $0,112^{*}$ & $0,131^{*}$ & $0,192^{*}$ & 0,232 \\
Out & 0,126 & 0,101 & $0,113^{*}$ & 0,138 & $0,050^{*}$ & $0,067^{*}$ & $0,129^{*}$ & $0,152^{*}$ \\
Nov & $0,064^{*}$ & $0,066^{*}$ & $0,106^{*}$ & 0,122 & $0,085^{*}$ & $0,110^{*}$ & $0,116^{*}$ & $0,137^{*}$ \\
Dez & $0,080^{*}$ & 0,114 & $0,064^{*}$ & $0,094^{*}$ & $0,058^{*}$ & $0,082^{*}$ & $0,078^{*}$ & $0,100^{*}$ \\
\hline
\end{tabular}

* significativo a $5 \%$

Na Figura 1, estão representados os valores de evapotranspiração de referência calculados para um período acumulado de 15 dias com diferentes níveis de probabilidade para os 12 meses do ano. Observa-se que a maior demanda hídrica foi encontrada para o mês de outubro com valores máximos de 11,86 e 11,71 $\mathrm{mm} \mathrm{dia}^{-}$ ${ }^{1}$ para a distribuição normal (A) e gama (B) com probabilidade de $95 \%$. Os menores valores foram observados para a probabilidade de 5\% apresentando 7,47 e $7,46 \mathrm{~mm} \mathrm{dia}^{-1}$ para a distribuição normal e gama. Possivelmente a ausência de chuva neste período contribuiu de maneira significativa para o aumento da ETo, fazendo com que a irrigação seja de grande importância nesta época. A adoção do nível de probabilidade para a ETo vai depender de diversos fatores incluindo as condições econômicas e o risco admissível do projeto de irrigação. DOOREMBOS \& PRUITT (1997) recomendam valores entre $75 \%$ e $80 \%$ nas condições de irrigação suplementar. SILVA et al. (2014) recomendam para o sertão cearense valores prováveis de ETo no período mensal para dimensionamento dos sistemas, enquanto ASSIS et al. (2014) sugerem os períodos de 10,15 e 20 dias para as probabilidades estudadas para este fim. 

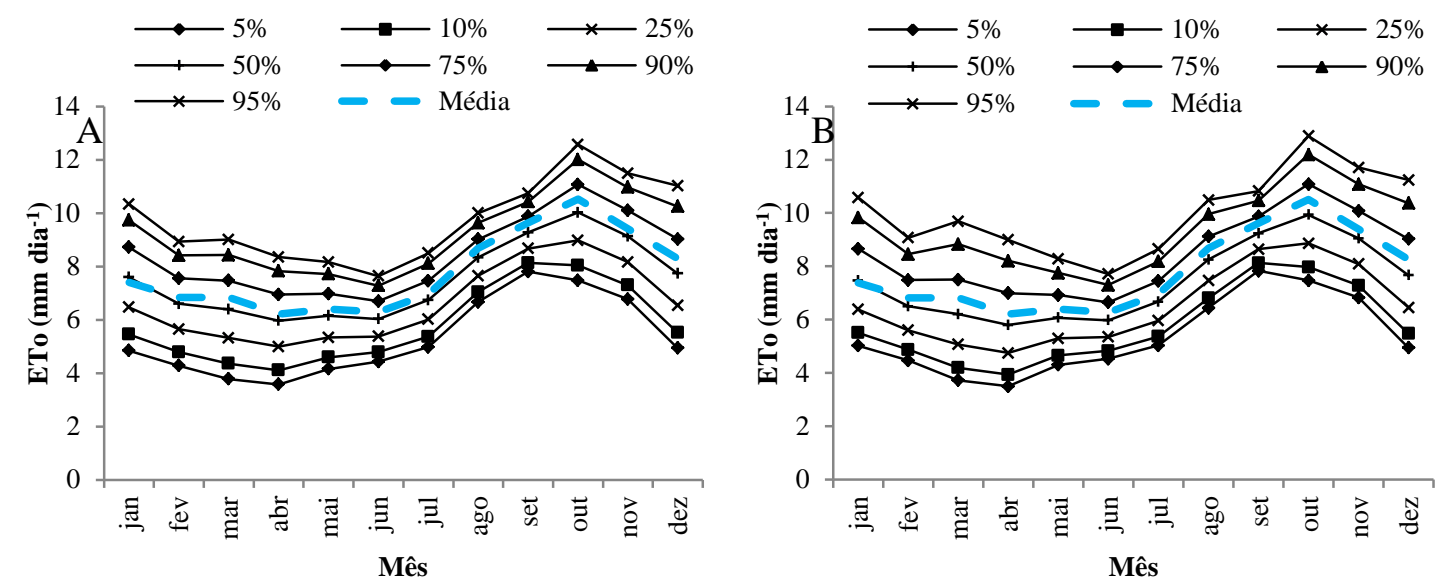

Figura 1. Evapotranspiração de referência calculada em intervalos de 15 dias com probabilidade de $5 \%, 10 \%, 25 \%, 50 \%, 75 \%, 90 \%$ e $95 \%$ utilizando a distribuição normal (A) e gama (B) para Petrolina-PE.

A Figura 2 apresenta a evapotranspiração de referência para os diferentes níveis de probabilidade de ocorrência para a distribuição normal em função da duração dos períodos estudados para o mês de outubro. Observa-se que houve uma diminuição dos valores da ETo com o aumento do período estudado. Com relação à probabilidade a ser utilizada para o uso em projetos de irrigação, diversos trabalhos como BACK (2007), SAAD et al. (2002) e FIETZ et al. (1997) recomendam que o valor da evapotranspiração de referência para o dimensionamento de sistemas de irrigação, seja obtido com $75 \%$ de frequência no período de maior demanda de água do ano, para esta probabilidade os períodos acumulados de $10, \quad 15$ e 30 dias (significativos pelo teste de aderência) apresentaram uma ETo de 10,7; 11,06 e $10,64 \mathrm{~mm} \mathrm{dia}{ }^{-1}$, sendo estes valores elevados quando comparados aos obtidos por SILVA et al. (1998) para a região de Cruz das Almas, BA.

Os valores observados para a distribuição gama (Figura 3) foram semelhantes aos observados na distribuição normal $(+0,34 \%$, em média) podendo ser ambas as distribuições utilizadas para a determinação da frequência da ETo. Para os períodos acumulados de 10, 15 e 30 dias a distribuição gama apresentou ETo de 10,7, 11,07 e 10,69 $\mathrm{mm}$ dia $^{-1}$ para a probabilidade de $75 \%$. 


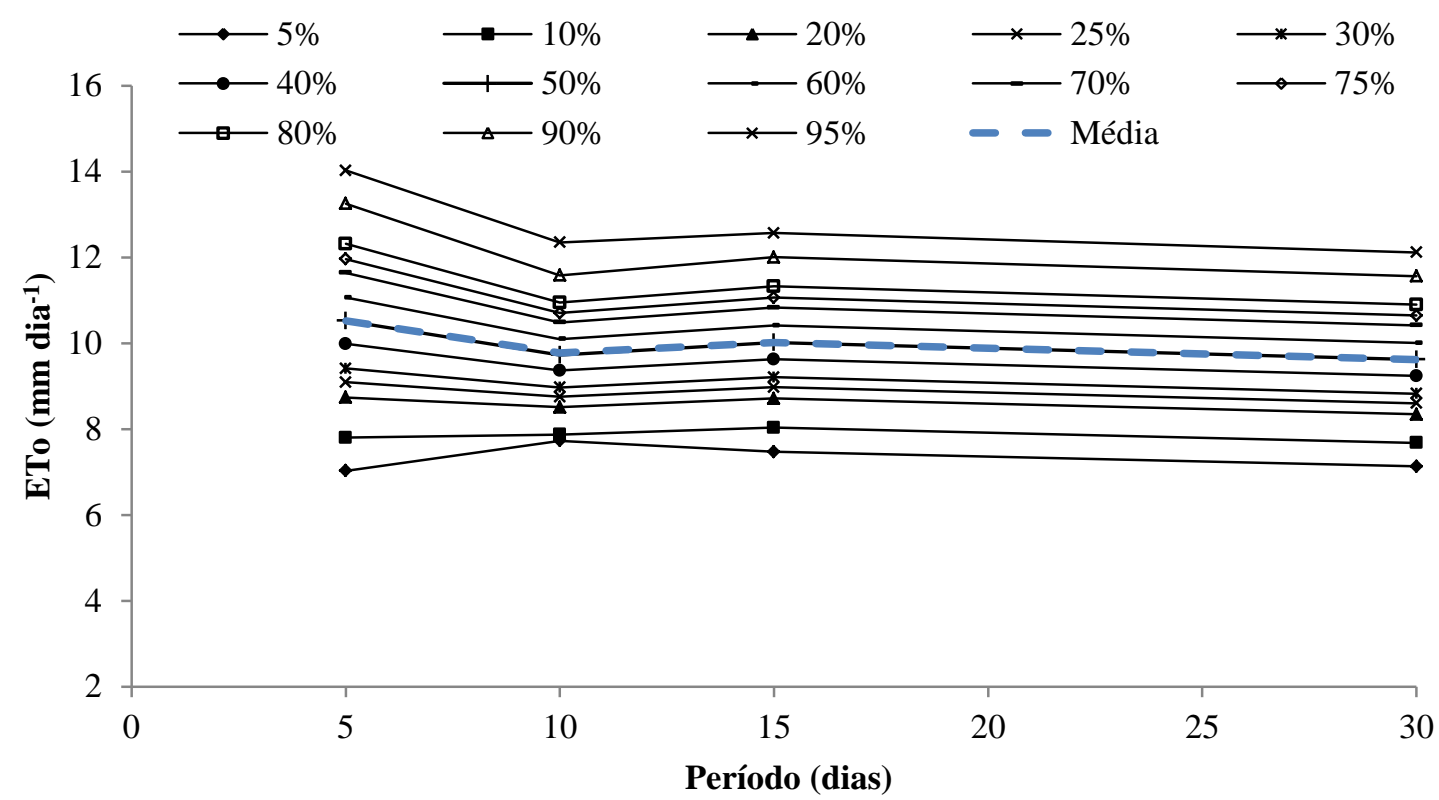

Figura 2. Evapotranspiração de referência para os diferentes níveis de probabilidade de ocorrência para a distribuição normal em função da duração do período para o mês de outubro.

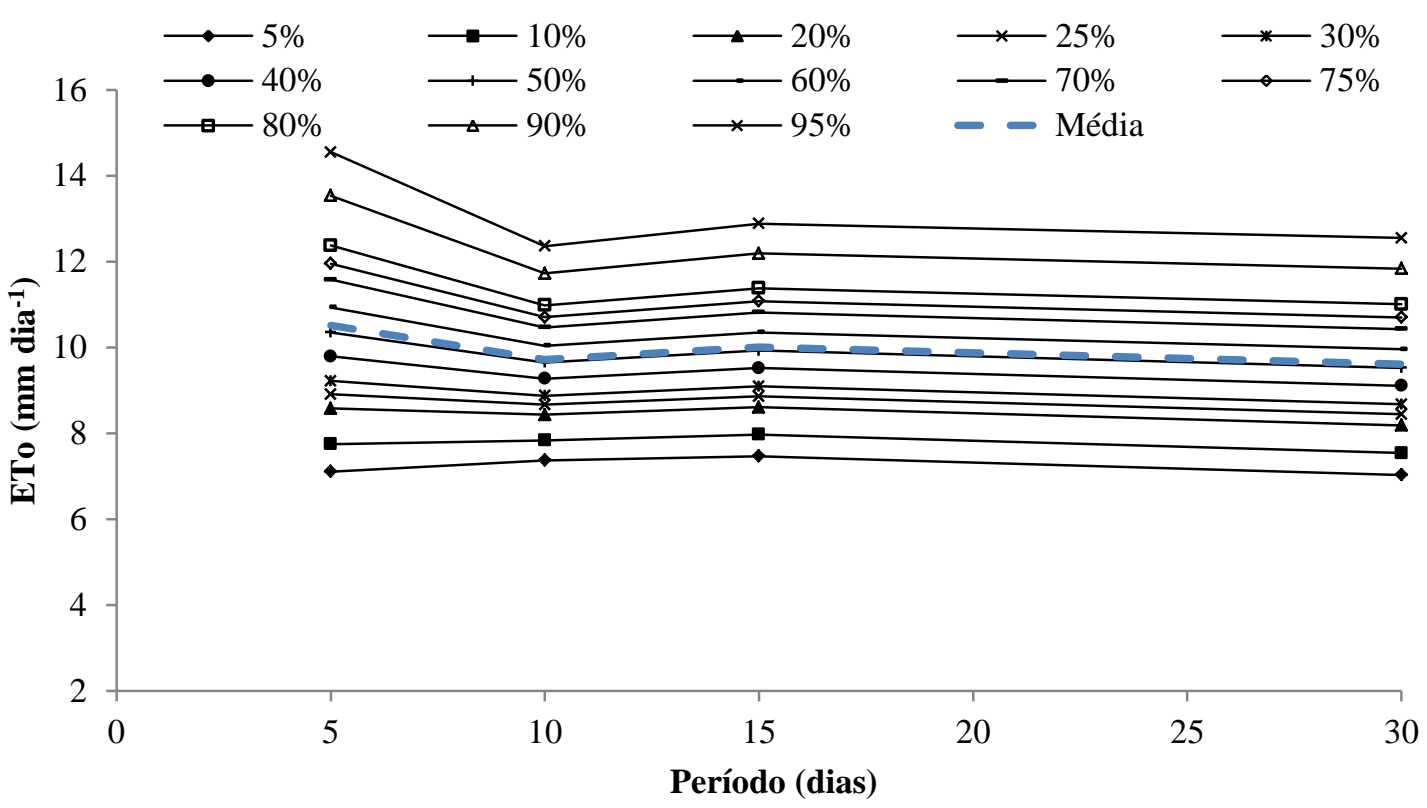

Figura 3. Evapotranspiração de referência para os diferentes níveis de probabilidade de ocorrência para a distribuição gama em função da duração do período para o mês de outubro.

\section{CONCLUSÃO}

As distribuições normal e gama foram aptas para representar os dados de evapotranspiração de referência para os períodos acumulados de 10,15 e 30 dias. A evapotranspiração de referência apresentou tendência de redução de acordo com o aumento do período acumulado, sendo recomendado o período acumulado de 15 dias com probabilidade de $75 \%$ para o dimensionamento de sistemas de irrigação para a região de Petrolina-PE. 


\section{REFERÊNCIAS BIBLIOGRÁFICAS}

ABABAEI, B. Are weather generators robust tools to study daily reference evapotranspiration and irrigation requirement? Water Resource Management, v. 28(1): 915-932, 2014.

ARAÚJO, E. M.; SILVA, I. N.; OLIVEIRA, J. B.; CAVALCANTE JÚNIOR, E. G.; ALMEIDA, B. M. Aplicação de seis distribuições de probabilidade a séries de temperatura máxima em Iguatu-CE. Revista Ciência Agronômica, v.41(1): 36-45, 2010.

ASSIS, J. P.; SOUSA, R. P.; BEZERRA NETO, F.; LINHARES, P. C. Tables of probabilities of reference evapotranspiration for the region of Mossoró RN, Brazil. Revista Verde, v.9 (3): 58-67, 2014.

BACK, A. J. Variação da evapotranspiração de referência calculada em diferentes intervalos de tempo. Engenharia Agrícola, v. 27 (1):139-145, 2007.

CARVALHO, D. F.; OLIVEIRA, L. F. C. Planejamento e manejo da água na agricultura irrigada. Viçosa: Ed. UFV, 2012, 240p.

CAMPOS, H. Estatística experimental não paramétrica. 4 ed. Piracicaba: Escola Superior de Agricultura Luiz de Queiroz, USP, 1983. 349p.

CHICHEPORTICHE, R.; BOUCHAUD, J. P. Weighted Kolmogorov-Smirnov test: accounting for the tails. Physical Review, v. 86 (4): 1539-3755, 2012.

CURTIS, F. Microsoft Excel 2010: passo a passo. Porto Alegre: Ed. Bookman, 2012, 400p.

DOORENBOS, J.; PRUITT, W.O. Crop water requirements. Roma: FAO, 1997. $212 p$.
FRIZZONE, J. A.; ANDRADE JÚNIOR, A. S. Planejamento de irrigação: Análise de decisão e investimentos. Brasília: Embrapa Informação Tecnológica, 2005. 626p.

FIETZ, C. R.; FRIZZONE, J. A.; FOLEGATTI, M. V.; PINTO, J. M. Probabilidade de ocorrência da evapotranspiração de referência na região de Dourados, MS. Ciência Rural, v. 27(2): 207-210, 1997.

PEIXOTO, D. T. C.; LEVIEN, S. L. A.; BEZERRA, A. H. F.; SOBRINHO, J. E. Avaliação de diferentes metodologias de estimativa da ETo baseadas no tanque classe A em Mossoró, RN. Revista Caatinga, v. 27 (2): 58-65, 2014.

SAAD, J. C.; BISCARO, G. A.; DELMANTO JÚNIOR, O.; FRIZZONE, J. A. Estudo da distribuição da evapotranspiração de referência visando o dimensionamento de sistemas de irrigação. Irriga, v. 7(1): 10-17, 2002.

SILVA, F. C.; FIETZ, C. R.; FOLEGATTI, M. V.; PEREIRA, F. A. C. Distribuição e frequência da evapotranspiração de referência de Cruz das Almas, BA. Revista Brasileira de Engenharia Agrícola e Ambiental, v.2(3): 284-286, 1998.

SILVA, A. P. N.; MONTENEGRO, A. A. A.; MOURA, G. B. A.; SILVA, J. J. N.; SOUZA, L. R. Chuva mensal provável para o Agreste de Pernambuco. Revista Brasileira de Ciências Agrárias, v. 8 (2): 287-296, 2013.

SILVA, A. P. N.; LIMA, F. J. L.; SILVA, A. O.; MOURA, G. B. A. Valores efetivos de precipitação pluvial para manejo da irrigação na cana-de-açúcar em Goiana, Pernambuco. Revista Brasileira de Ciências Agrárias, v.5 (4): 585-591, 2010. 
SILVA, M. G.; BATISTA, L. S.; CARMO, F. F.; SANTOS, N. T.; OLIVEIRA, I. S. Probabilidade de ocorrência de valores estimados de evapotranspiração de referência no Sertão do Ceará. Engenharia na Agricultura, v. 22 (6): 562-574, 2014.
THOM, H. C. S. A note on the gamma distribution. Monthly Weaher Review, v.86(4): 117-122, 1958.

TRAJKOVIC, S.; KOLAKOVIC, S. Comparison of simplified pan-based equations for estimating reference evapotranspiration. Journal of Irrigation and Drainage Engineering, v. 136, 137140, 2010. 